\title{
Title: Textual Analysis of General Surgery Residency Personal Statements: Topics and Gender Differences
}

Authors: Laura Ostapenko $\mathrm{MD}^{1}$, Cheryl Schonhardt-Bailey, $\mathrm{PhD}^{2}$, Jessica Walling Sublette $\mathrm{BA}^{3}$, Douglas S. Smink $\mathrm{MD}^{1}$ and Nora Y. Osman $\mathrm{MD}^{3}$

Forthcoming in Journal of Surgical Education, (2018 [September/October], 75, 5)

From: ${ }^{1}$ The Department of Surgery, Brigham \& Women's Hospital and Harvard Medical School, Boston, MA, ${ }^{2}$ The London School of Economics and Political Science, London, England, and ${ }^{3}$ The Department of Medicine, Brigham \& Women’s Hospital and Harvard Medical School, Boston, MA

Highlights:

- Analyzed the ERAS personal statements of 578 applicants from LCME-accredited medical schools to a single ACGME-accredited general surgery program

- Identified and labeled eight statistically significant themes through independent review: "my story,” "the art of surgery,” "clinical vignettes,” "why I love surgery,” "residency program characteristics," "working as a team,” "academics and research,” and "global health and policy"

- Identified gender-specific differences: women were significantly more likely than men to be represented within the theme of "working as a team," while men were significantly more likely than women to be represented within the theme of "clinical vignettes" 


\section{Corresponding Authors:}

Laura Ostapenko, MD

Address all reprint requests to:

Laura Ostapenko, MD

Brigham and Women's Hospital

75 Francis Street, Boston, MA 02115

lostapenko@partners.org

617-732-5500

Keywords: Medical Student, Surgery Residency, Personal Statement, Textual Analysis

Submitted: June 20, 2017

Resubmitted: September 6, 2017

Abstract word count: 289

Tables: 2

Figure: 1

Appendix: 1 
Title: Textual Analysis of General Surgery Residency Personal Statements: Topics and Gender Differences

Purpose: Applicants to US general surgery residency training programs submit standardized applications. Applicants use the personal statement to express their individual rationale for a career in surgery. Our research explores common topics and gender differences within the personal statements of general surgery applicants.

Methods: We analyzed the ERAS personal statements of 578 applicants (containing 382405 words) from LCME-accredited medical schools to a single ACGME-accredited general surgery program using an automated textual analysis program to identify common topics and gender differences. Using a recursive algorithm, the program identified common words and clusters, grouping them into topic classes which are internally validated.

Results: We identified and labeled eight statistically significant topic classes through independent review: "my story," "the art of surgery," “clinical vignettes," "why I love surgery," "residency program characteristics," "working as a team," "academics and research,” and "global health and policy.” Although some classes were common to all applications, we also identified genderspecific differences. Notably, women were significantly more likely than men to be represented within the class of "working as a team." $(\mathrm{p}<0.01)$ Furthermore, men were significantly more likely than women to be represented within the class of "clinical vignettes.” $(p<0.01)$

Conclusions: Applying textual analysis to a national cohort, we identified common narrative topics in the personal statements of aspiring general surgeons, noting differences between the statements of men and women. Women were more likely to discuss surgery as a team endeavor while men were more likely to focus on the details of their surgical experiences. Our work mirrors what has been found in social psychology research on gender-based differences in how men and 
women communicate their career goals and aspirations in other competitive professional situations. 
Introduction

In the United States (US), medical students embark on the path from medical school to residency by submitting a standardized application to selected residency programs through the Association of American Medical Colleges (AAMC) Electronic Residency Application Service (ERAS). The application consists of transcripts, letters of recommendation, a “Dean's letter” or Medical Student Performance Evaluation (MSPE), extracurricular activity descriptions, a curriculum vitae (CV), and a personal statement. ${ }^{1}$

The composition of personal statements is not standardized. The ERAS application provides no official guidelines on writing a personal statement. ${ }^{2}$ However, advice abounds. University sites, academic office websites and on-line personal statement warehouses offer advice, counseling, and fee-for-service editing. ${ }^{3}$ The AAMC Careers in Medicine website advises applicants to write personal statements that meet three broad goals: communicate enthusiasm for and dedication to your specialty; address potential reviewer concerns; and provide topics for discussion during interviews. $^{4}$

Just as the writing of personal statements is not standardized, there is no coordinated or standardized use of personal statements by residency programs. Surveys of residency program directors demonstrate wide variation in the use of personal statements in the resident selection process. ${ }^{5-8}$ One reason for this wide variation may be that characteristics of a high quality personal statement are unclear. Research has shown that there is low inter-rater reliability when assessing personal statement quality. ${ }^{6}$

The lack of standardization in the writing and use of personal statements gives personal statements a uniquely personal status within the ERAS application. Personal statements allow applicants to choose how best to communicate their rationale and aspirations for their career to their chosen professional community. The necessity of choice makes personal statements a rich data source. 
Previous studies examining the role of personal statements in the application process have used human readers to identify a few specialty-specific topics within personal statements, specifically in Anesthesiology. ${ }^{5}$ Recently, we showed that large-scale textual analysis allows an exhaustive characterization of the significant topics within large cohorts of specialty-specific personal statements. Furthermore, we identified subtle differences between male and female cohorts in the topics they employed to communicate their aspirations and career goals. ${ }^{9}$

Based on our previous work, we hypothesized that a large-scale textual analysis could identify significant topics in the personal statements of applicants to General Surgery residency programs. We further hypothesized that such an analysis would allow us to identify significant differences between men and women in the topics each employed to communicate their aspirations and career goals. In this manuscript, we describe a large-scale textual analysis of the personal statements of general surgery applicants, noting both the common topics and the gender differences.

\section{Methods}

\section{Study Population}

We collected the personal statements submitted to ERAS in the applications for post-graduate training at Brigham and Women’s Hospital for academic year 2013-14. We included all applicants from Liaison Committee on Medical Education (LCME)-accredited US or Canadian medical schools applying to post-graduate training programs at the Brigham and Women's Hospital in General Surgery during the academic year 2013-2014 in the analysis. This study was approved by the Partners Health Care Institutional Review Board.

\section{Data Collection and Analysis}

We performed textual analysis of the corpus using Alceste® (ALCESTE VERSION 2012 ENTERPRISE, Image-Zafar.com, Toulouse, France) an independent contextual analysis software program previously described. ${ }^{9}$ Our method was dictated in part by the algorithms of Alceste. 
To prepare the personal statements for analysis, we de-identified, numbered, and collated all the personal statements in a single document, termed a 'corpus'. We then standardized certain words within the corpus for the sake of consistency and efficiency. For example, we replaced words "brother" and "sister" with the word "sibling," and standardized personal nouns such as "Mr. Smith.” We then analyzed this corpus using Alceste.

Alceste categorizes text into segments called 'elementary contextual units' (ECUs). Each ECU is a sentence or a chunk of sentences that represents an individual unit of meaning. ECUs are both identified and grouped into classes based on statistical word co-occurrence. For example, in our corpus, "competition" is often found in close proximity to "sport." Furthermore, "sport" is often found in close proximity to "athlete." These branching relationships between words allow Alceste to identify and group ECUs into classes. These word co-occurrences and the relationships that they suggest can be visualized with a tree diagram (or dendrogram). Tree diagrams for two of the classes are provided in the Appendix. Alceste uses word co-occurrence to generate classes that are both as internally homogenous and as externally distinct from the other classes as possible.

The end product of this analysis for the corpus as a whole is threefold. First, Alceste produces a hierarchy of classes or dendrogram. Within each class, Alceste also ranks the ECUs and the words that make up that class according to statistical significance, each ranked in order by phi coefficient and $\chi^{2}$ statistical significance, with the minimum $\chi^{2}$ for a classified word equaling 18.25 , with one degree of freedom. Finally, Alceste provides a tree diagram the displays the relationship between words within each class.

We independently and blindly reviewed the completed statistical output. We reviewed all ECUs and characteristic words for each of the topic classes, and provided a blinded interpretation of the topic of each class. We then conferred and agreed upon descriptive labels for each class. The descriptive label for each class is based on both ECUs and on the most characteristic words for that class. 
We also conducted a separate (“cross-data”) gender analysis by tagging the personal statements written by men and women. Within the corpus, Alceste is able to use the tags to divide the aggregate personal statements written by women from the aggregate personal statements written by men. Looking at each group of aggregate personal statements, Alceste identifies statistically significant differences in the frequency and weight of class occurences in one group compared to another group. Alceste reports whether a statistically significant difference in class occurences is present. We then quantified the difference between men and women for each of the classes by counting the number of significant ECUs within each class for each gender. This can be found in Table 1. Because of the specific algorithms of Alceste, our analysis does not include data related to individual personal statements, focusing rather on gender differences found in the group as a whole.

Results

We analyzed 578 personal statements submitted by medical students from LCME-accredited medical school to the Brigham and Women's Hospital General Surgery Residency Program during academic year 2013-2014. 44\% of the applicants were women, 56\% were men. The greater number of male applicants reflects the greater number of male applicants, as observed in other studies of general surgery applicant characteristics. ${ }^{10}$ The full edited corpus contained 382,405 words.

We performed textual analysis of the corpus using Alceste's internal algorithm in an unsupervised manner without pre-coding or pre-selection. The program successfully classified $74 \%$ of the corpus into statistically significant classes. The remaining text could not be classified by the software program and was therefore excluded from the analysis. Alceste identified 8 statistically different topic classes. Using the ECUs and characteristic words for each class, we describe the following interpretations of those eight classes, with percentage weights in brackets: 
1. My story (22\% of the classified ECUs): This class is characterized by vivid accounts of family or childhood, often focusing on overcoming challenges, or the inspiration to pursue medicine.

2. Working as a team (15\%): This class is made up of descriptions of teams - from clinical teams to sports teams.

3. The art of surgery (13\%): This class focuses on the technical aspects of surgery, highlighting the precision, efficiency, and meticulousness of surgery.

4. Clinical vignettes (12\%): This class describes specific patients, operations, and clinical scenarios.

5. Why I love surgery (8\%): This class focuses on the field of general surgery and describes the special draw of general surgery when compared to surgical subspecialties and medical specialties.

6. Residency program characteristics (14\%): This class is characterized by descriptions of what applicants are seeking from a residency as well as what applicants can bring to their residencies.

7. Academics and research (9\%): This class focuses on achievement in scholarship and research.

8. Global health and policy (7\%): This class highlights accomplishments in global health - both through service and through organizations.

Key words and examples of representative ECUs for each class are shown in Table 2.

The analysis allows us to ascertain whether key indicators (independent of the text itself), such as gender, are statistically associated with any of the thematic classes. Notably, women were more likely than men to be represented within the class of working as a team $(p<0.01)$, while men were 
more likely than women to be represented within the class of clinical vignettes. $(\mathrm{p}<0.01)$ Figure 1 shows the dendrogram for the analysis, in which the degree of co-occurrence (or clustering) between the classes is illustrated. Of the eight classes, only two obtain statistical significance by gender, as indicated. The remaining classes are not distinct to either gender.

\section{Discussion}

The personal statement is the most personal element of the residency application. Yet, by aggregating personal statements of applicants in the same field, we can examine the common topics applicants use to communicate their aspirations and career goals. In this manuscript, we describe a large-scale textual analysis of the personal statements of 578 applicants to a single general surgery residency program. Based on our previous analysis of the personal statements of a large internal medicine cohort, we hypothesized that we would find common topics and genderspecific differences in how general surgery applicants communicate their career goals and aspirations. ${ }^{9}$

In our analysis, we found that the large majority of content in the personal statements of general surgery applicants can be categorized into 8 classes. The general classes that we identified can be divided into the categories of personal (“my story”), academic (“academics and research,” “global health and policy”), educational ("residency program characteristics”), and clinical (“working as a team,” "the art of surgery," “clinical vignettes”). Based on previous work, we expected to find these topics. ${ }^{5,9}$ Other researchers have found medical students express similar rationale when explaining their choice of surgery as a career. ${ }^{11}$ Furthermore, we expected these topics to be realized through language and examples specific to surgery - such as the class "the art of surgery" or surgical "clinical vignettes.”

While all of the classes were represented in both the male and female cohorts, we identified genderspecific differences in the classes of "working as a team" and "clinical vignettes." Women were 10 
significantly more likely than men to be represented within the class of "working as a team" while men were significantly more likely than women to be represented within the class of "clinical vignettes."

Within the class of "working as a team," applicants often discussed their previous experiences with teams sports, such as in the following representative quotation: "[P]laying team sports has taught me the importance of collaboration and interpersonal communication for communal achievement." A characteristic example of the class of "clinical vignette" tells the story of a student's encounter with a patient: "On the first day of my general surgery rotation, I helped transfer [the patient] onto the operating room table. After induction and intubation, we removed the sheets from his body and prepped his abdomen for the procedure: an exploratory laparotomy for relief of small bowel obstruction." The differences in the representation of women and men within these classes reflects a difference in how men and women chose to communicate their career goals and aspirations.

Within surgery, gender differences in leadership positions, professional awards, and other areas have been well documented. ${ }^{12,13}$ There is also a robust social sciences literature on gender differences in professional settings, motivated by similar gender differences in achievement across many professions. The social science literature has produced three main groups of explanatory theories to explain these gender differences. First, some researchers have hypothesized that gender differences in professional accomplishments are explained by differences in perferences. ${ }^{14}$ Other researchers have hypothesized that gender differences in professional success are explained by differences in behavior. ${ }^{15-17}$ Still other researchers have examined the impact of institutional and societal structures on both preferences and behaviors. ${ }^{18-20}$

While there are many possibilities to explain the gender differences we observed, we put forward two leading hypotheses. First, the gender differences we observed may reflect differences in actual motivating factors for career goals and aspirations. A second possibility is these gender differences may reflect differences in perceptions of what makes a successful General Surgery personal statement. Given our method, we cannot evaluate whether the gender-specific differences we 11 
observe in the classes reflect differences in actual career goals and aspirations. As a result, we will focus our discuss on possible explanations for why men and women would choose different ways to communicate their preferences. With reference to well-substantiated social sciences research, we hypothesize that institutional and societal structures may be affecting the ways men and women communicate their preferences. Specifically, a robust body of social science literature suggests that successful communication strategies in competitive application processes look different for men and women.

In competitive professional situations, both men and women need to self-promote to appear competent. While men are often rewarded for self-promotion, women are often penalized for selfpromotion, as self-promotion runs contrary to gender normative stereotypes of women as modest. ${ }^{21-24}$ Women who self-promote are perceived as less nice and more demanding than either men who self-promote or women who self-efface. ${ }^{25,} 26$ If women do self-promote, they are more likely to be perceived as competent but less likely to be successful in competitive professional situations. ${ }^{21}$ This research from the social sciences on gender-specific differences in selfpromotion provides one possible explanation for the gender-specific differences in the topics that emerge in the personal statements of surgery applicants.

The social science research finding that men are more likely to create a compelling narrative through individual self-promotion provides one hypothesis for our finding that men are more likely to be represented within the class of "clinical vignettes." In this class, applicants discuss their experience as individuals with specific patients, operations, and scenarios, such as in the following representative quotation:

It was 3:00 a.m. and I had been awake for 24 hours and counting. I spent the entire day in the operating rooms of the burns hospital, followed by trauma call in the evening. A patient with diverticulitis and a perforated colon was rushed to the operating room for an exploratory laparotomy; excited to scrub in, I willingly volunteered. 
The author of this vignette engages in individual self-promotion: describing a clinical scenario from a first-person perspective, highlighting dedication to and enthusiasm for clinical care.

While women are constrained in their ability to self-promote by the gender normative stereotype of women as modest, women can appear both collegial and competent if they self-promote by offering an account of their accomplishments that focuses on their relationships with others. ${ }^{22,} 25$ By focusing on their work in relation to a team, women can discuss their accomplishments without incurring a backlash for appearing immodest.

The social science research finding that women are more likely to create a compelling narrative by focusing on team success provides one hypothesis to explain our finding that women were more likely than men to be represented within the class of working as a team. In this class, applicants discuss their experiences leading and supporting colleagues. In the following quotation, an applicant discusses success in the context a team:

As a lifelong player and captain of my collegiate varsity team I developed the drive and commitment to stay focused on a goal through challenges and sacrifices similar to the dedication surgeons have for their craft. Through my years of athletics I also came to learn that success is the result of team efforts. As team captain, I refined my ability to motivate others to achieve a common goal and reach their highest individual potential.

The author of this quotation self-promotes by highlighting a previous leadership role ("captain”) along with desirable personal characteristics (“drive” and "commitment”). However, this selfpromotion happens within the context of a team. The author emphasizes the importance of others in personal success by defining success as a "common goal" and "the result of team efforts."

Even if the gender-specific differences are explained by different perceptions of what makes a successful personal statement, it is unlikely that the different strategies employed by men and women are deliberate. The applicants may not be aware of the thematic choices they are making. 13 
Men and women could be more likely to choose certain topics because they have successfully used these topics in the past or because they received positive feedback on their use of these topics in their current personal statement.

\section{Limitations}

We acknowledge certain limitations to our investigation. The first is the subjective bias that is inherent to the use of computer software programs in textual analysis. By blindly and independently interpreting the output, we sought to mitigate some of this subjectivity. We also used a program that has been extensively studied in the social sciences and recently proven valuable in the medical literature. We acknowledge, however that there remains some subjectivity to the interpretation of this textual analysis.

Another limitation related to our textual analysis lies in the residual text that the computer program cannot classify and therefore excludes from the analysis. Our data set includes $74 \%$ of the surgery corpus, meaning leaving $26 \%$ unclassified. There may be some less common topics raised by a small number of applicants which go unidentified because the prevalence of word co-occurrence is too small to classify. Likewise the language used to describe less significant topics may be more diverse, subtle or nuanced, making it unrecognizable or unclassifiable for the program we used.

Finally, we acknowledge that our study cohort included only applicants from LCME-accredited medical schools to a single US training program. However, with nearly $75 \%$ of the total US surgery pool, we believe that our data set was sufficiently robust for analysis, and representative of the typical US medical student applicant to General Surgery residency programs. ${ }^{27}$ We acknowledge that such data may be difficult to translate outside the North American training paradigm. 


\section{Conclusion}

Through the aggregate textual analysis of a large number of personal statements written by surgery residency applicants, we describe common topics and gender-specific differences in how applicants communicate their career goals and aspirations. Our findings of gender-specific differences could hold practical significance for program directors while assessing male and female applicants. A deeper understanding of the differences may also allow program directors to harness these trends to build stronger, more diverse and truly inclusive residency programs.

1. American Association of Medical Colleges. About ERAS ${ }^{2} 2017$.

2. American Association of Medical Colleges. MyERAS Personal Statements. 2017.

3. eras personal statement advice - Google Search2017.

4. Tysinger J, Nadeau MT. Writing your personal statement - Applying - Residency Application - Prepare for Residency - Careers In Medicine2017.

5. Max BA, Gelfand B, Brooks MR, Beckerly R, Segal S. Have personal statements become impersonal? An evaluation of personal statements in anesthesiology residency applications. Journal of clinical anesthesia. 2010;22:346-351.

6. White BA, Sadoski M, Thomas S, Shabahang M. Is the evaluation of the personal statement a reliable component of the general surgery residency application? Journal of surgical education. 2012;69:340-343.

7. Ferguson E, James D, O'Hehir F, Sanders A, McManus IC. Pilot study of the roles of personality, references, and personal statements in relation to performance over the five years of a medical degree. BMJ. 2003;326:429-432.

8. Crane J, Ferraro C. Selection Criteria for Emergency Medicine Residency Applicants. Academic Emergency Medicine. 2000;7:54-60.

9. Osman NY, Schonhardt-Bailey C, Walling JL, Katz JT, Alexander EK. Textual analysis of internal medicine residency personal statements: themes and gender differences. Med Educ. 2015;49:93-102.

10. Stain SC, Hiatt JR, Ata A, et al. Characteristics of highly ranked applicants to general surgery residency programs. JAMA Surg. 2013;148:413-417.

11. Marshall DC, Salciccioli JD, Walton SJ, Pitkin J, Shalhoub J, Malietzis G. Medical student experience in surgery influences their career choices: a systematic review of the literature. $J$ Surg Educ. 2015;72:438-445.

12. Cochran A, Freischlag JA, Numann P. Women, surgery, and leadership: where we have been, where we are, where we are going. JAMA Surg. 2013;148:312-313.

13. Sexton KW, Hocking KM, Wise E, et al. Women in academic surgery: the pipeline is busted. J Surg Educ. 2012;69:84-90. 
14. Gino F, Wilmuth CA, Brooks AW. Compared to men, women view professional advancement as equally attainable, but less desirable. Proc Natl Acad Sci U S A. 2015;112:1235412359.

15. Buser T, Niederle M, Oosterbeek H. Gender, Competitiveness, and Career Choices. The Quarterly Journal of Economics. 2014;129:1409-1447.

16. Eagly AH, Steffen VJ. Gender and aggressive behavior: a meta-analytic review of the social psychological literature. Psychol Bull. 1986;100:309-330.

17. LaFrance $M$, Hecht MA, Paluck EL. The contingent smile: a meta-analysis of sex differences in smiling. Psychol Bull. 2003;129:305-334.

18. Bowles HR, Babcock L, Lai L. Social incentives for gender differences in the propensity to initiate negotiations: Sometimes it does hurt to ask. Organizational Behavior and Human Decision Processes. 2007;103:84-103.

19. Heilman ME, Eagly AH. Gender Stereotypes Are Alive, Well, and Busy Producing Workplace Discrimination.

20. Eagly AH, Karau SJ. Role congruity theory of prejudice toward female leaders. Psychol Rev. 2002;109:573-598.

21. Rudman LA. Self-promotion as a risk factor for women: the costs and benefits of counterstereotypical impression management. J Pers Soc Psychol. 1998;74:629-645.

22. Amanatullah ET, Morris MW. Negotiating gender roles: gender differences in assertive negotiating are mediated by women's fear of backlash and attenuated when negotiating on behalf of others. J Pers Soc Psychol. 2010;98:256-267.

23. Costrich N, Feinstein J, Kidder L, Marecek J, Pascale L. When stereotypes hurt: Three studies of penalties for sex-role reversals. Journal of Experimental Social Psychology. 1975;11:520-530.

24. Maliniak D, Powers $\mathrm{R}$, Walter BF. The gender citation gap in international relations. International Organization. 2013;67:889-922.

25. Bowles HR, Babcock L. How Can Women Escape the Compensation Negotiation Dilemma? Relational Accounts Are One Answer. Psychology of Women Quarterly. 2013;37:8096.

26. Kolehmainen C, Brennan M, Filut A, Isaac C, Carnes M. Afraid of being "witchy with a 'b"': a qualitative study of how gender influences residents' experiences leading cardiopulmonary resuscitation. Acad Med. 2014;89:1276-1281.

27. Program NRM. Results and Data: 2013 Main Residency Match®. Washington, DC2013. 
Surgery Personal Statements

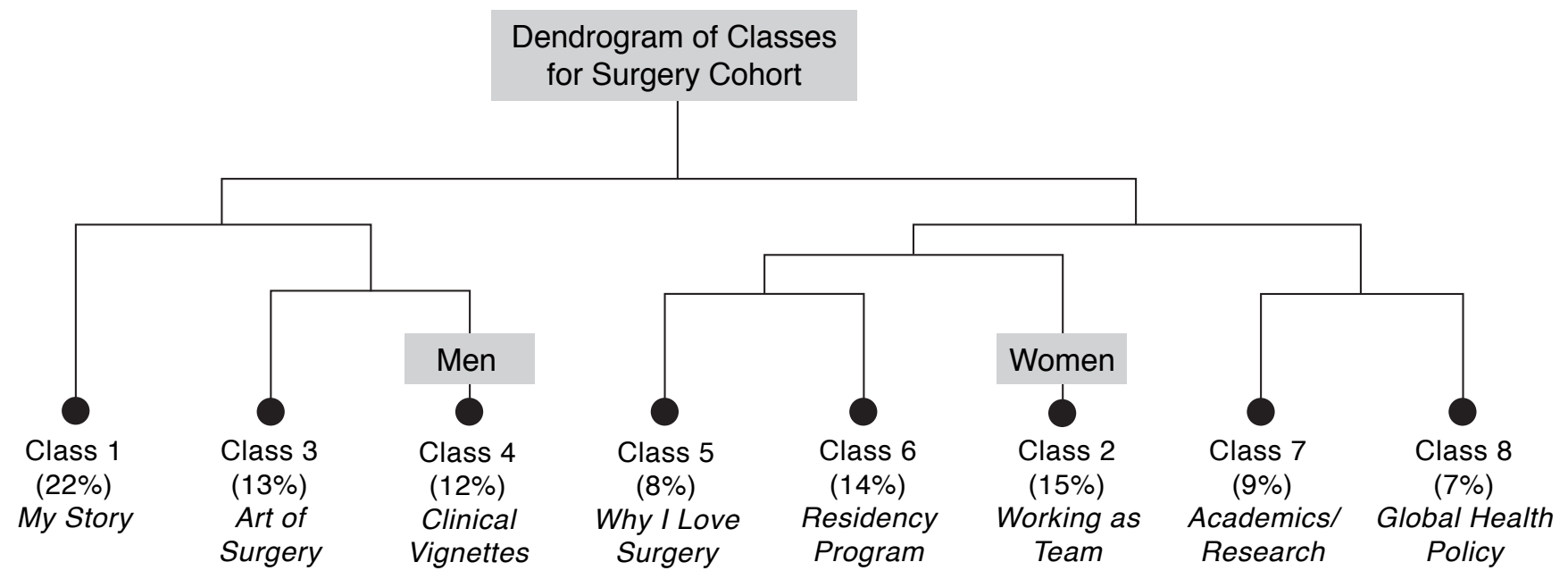

Figure 1: Dendrogram of Surgery Personal Statements 
Table 1: Summary of analyzed text and descriptions of the most common thematic classes in personal statements

written by general surgery residency applicants, AY 2013-2014

\section{Total word count}

Unique words analysed (freq $>3$ )

Minimum $\chi^{2}$ for word selection

Classified E.C.U.s
382,405

178,150

$-18$

$74 \%(5474)$

\section{Topic}

Classes

Title and Description (based on blinded and independent review)

1

My Story: This class is characterized by vivid accounts of family or childhood, often focusing on overcoming challenges, or the inspiration to pursue medicine.

2 Working as a Team: This class is made up of descriptions of teams - from clinical teams to sports teams.

The Art of Surgery: This class focuses on the technical aspects of surgery, highlighting the precision, efficiency, and meticulousness of surgery.

$4 \quad$ Clinical Vignettes: This class describes specific patients, operations, and clinical scenarios.

Why I Love Surgery: This class focuses on the field of general surgery and describes the special draw of general surgery when compared to surgical subspecialties and medical specialties.

Residency Program Characteristics: This class is characterized by descriptions of what applicants are seeking from a residency as well as what applicants can bring to their residencies.

$7 \quad$ Academics and Research: This class focuses on achievement in scholarship and research.

8

Global Health and Policy: This class highlights accomplishments in global health - both through service and through organizations.

\begin{tabular}{|c|c|c|}
\hline $\begin{array}{c}\text { Proportion of } \\
\text { all classified } \\
\text { ECUs }\end{array}$ & \multicolumn{2}{|c|}{$\begin{array}{c}\text { Most Representative } \\
\text { ECUs in Class by }\end{array}$} \\
\hline $22 \%$ & Men & Women \\
\hline $15 \%$ & 63 & 44 \\
\hline $13 \%$ & 33 & $52 * *$ \\
\hline $12 \%$ & 33 & 40 \\
\hline $8 \%$ & $39 * *$ & 26 \\
\hline $14 \%$ & $31 *$ & 15 \\
\hline $9 \%$ & 36 & 42 \\
\hline $7 \%$ & 24 & 25 \\
\hline
\end{tabular}

Gender tag $\chi^{2}$ statistical significance $(\mathrm{df}=1):{ }^{*} p<.05{ }^{* *} p<.01$ 
Table 2: Top-ranking words and ECUs from the identified thematic classes in personal statements written by General Surgery applicants.

Class

Key Words

\section{My story}

\section{Working as a \\ team}

The art of
surgery

Clinical

vignettes

up, father, mother, family,

sibling, grow, old, young, parent, people

team, hard, dedicate, ethic, teamwork, value, importance, persevere, self, work

anatomy, hand, dissect, feel, body, knot, human, suture, operating, art

trauma, abdominal, patient, icu, resect, repair, emergency department, abdomen, bowe important patient

field, problem, general

Why I love surgery

Residency

program

characteristics

Academics and

research

Global health and policy surgery, technical, solving, definitive, surgery, general, intellect, knowledge residency, program, look, academic, train, hope, research, future, provide, institution manuscript, study, author

\section{Representative ECU}

"Lucha por lo que quieras, Hija," my father would always tell me growing up. "Strive for want you want, Daughter." That phrase, which I have heard him say many times to my younger siblings and me, has served as my driving force.

Contributing to the success of the team in an unorthodox manner was a unique challenge, and I relished being an important part of our team's success despite sacrificing my personal desire to play. During my college softball career, I learned the importance of humility and the meaning of teamwork. I will be a great surgeon because I am a gym rat, a diligent worker, a good person, and an outstanding teammate.

watched in awe as the two attending doctors worked with precision and efficiency, gracefully anticipating each other's every move. I was truly amazed as, after a series of unbelievably intricate knots tied in delicate 7-0 prolene bound together tiny vessel walls...

During the course of a 5 hour operation, a chest tube is placed, multiple enterotomies are repaired, a segment of small bowel is resected, a cholecystectomy is performed, and the liver is packed. The patient is left in discontinuity with an open abdomen and transferred to the ICU with a plan to return to the operating room the following day.

General surgery uniquely covers the depth of pathologies, patients, challenges, and solutions that offers the most latitude to discover which area is truly suited to the individual while establishing a foundation of knowledge to care for the whole patient.

I would like to train somewhere where I will be exposed to a diverse patient population with a wide variety of clinical problems. I believe it is this kind of well-rounded, supportive, and diverse environment that will allow me to best apply my enthusiasm and motivation to become the best surgeon possible.

research, project, investigate, For my Master of Public Health thesis, I conducted community-based participatory research among focus groups of underserved data, clinical, publish, basic, patients with cancer in order to better identify patient-centered cancer delivery outcomes. The findings of these focus groups were used to inform an intervention design, which received grant funding from the Patient-Centered Outcomes Research Institute.

health, student, global, As someone who has been given much, I am deeply driven to give back. I have done so through tutoring students of grade school to underserved, healthcare, med, medical school, providing healthcare in free clinics to the urban and rural underserved, and helping to enhance public health efforts policy, president, school, tutor against HIV in Vietnam. 


\section{Appendix 1: Selected Tree Diagrams}

The following tree diagrams provide visual representations of how Alceste obtains classifications of words and ECUs. The vertical lines between plus signs show the word co-occurrences. The further toward the left the connection, the greater the co-occurrence is between the words.

Class 2 - Working as a team: This class is made up of descriptions of teams - from clinical teams to sports teams.

Overcome
Obstacle
Adapt
Situation
Challenge
Difficult
Mental
Diligence
One's
Balance
Demand
Patience
Professional
Personal
Sacrifice
Commit
Success
Ability
Communication
Thrive
Pressure
Leader
Importance
Teamwork
Member
Value
Learn
Team
Maintain
Football
Reliable
Coach
Individual
Teammate
Common
Achieve
Goal
Athlete
Athletic
Sport
Competition
Captain
Play
Soccer
Work
Hard
Value
Ethic
Succeed
Willingness
Detail

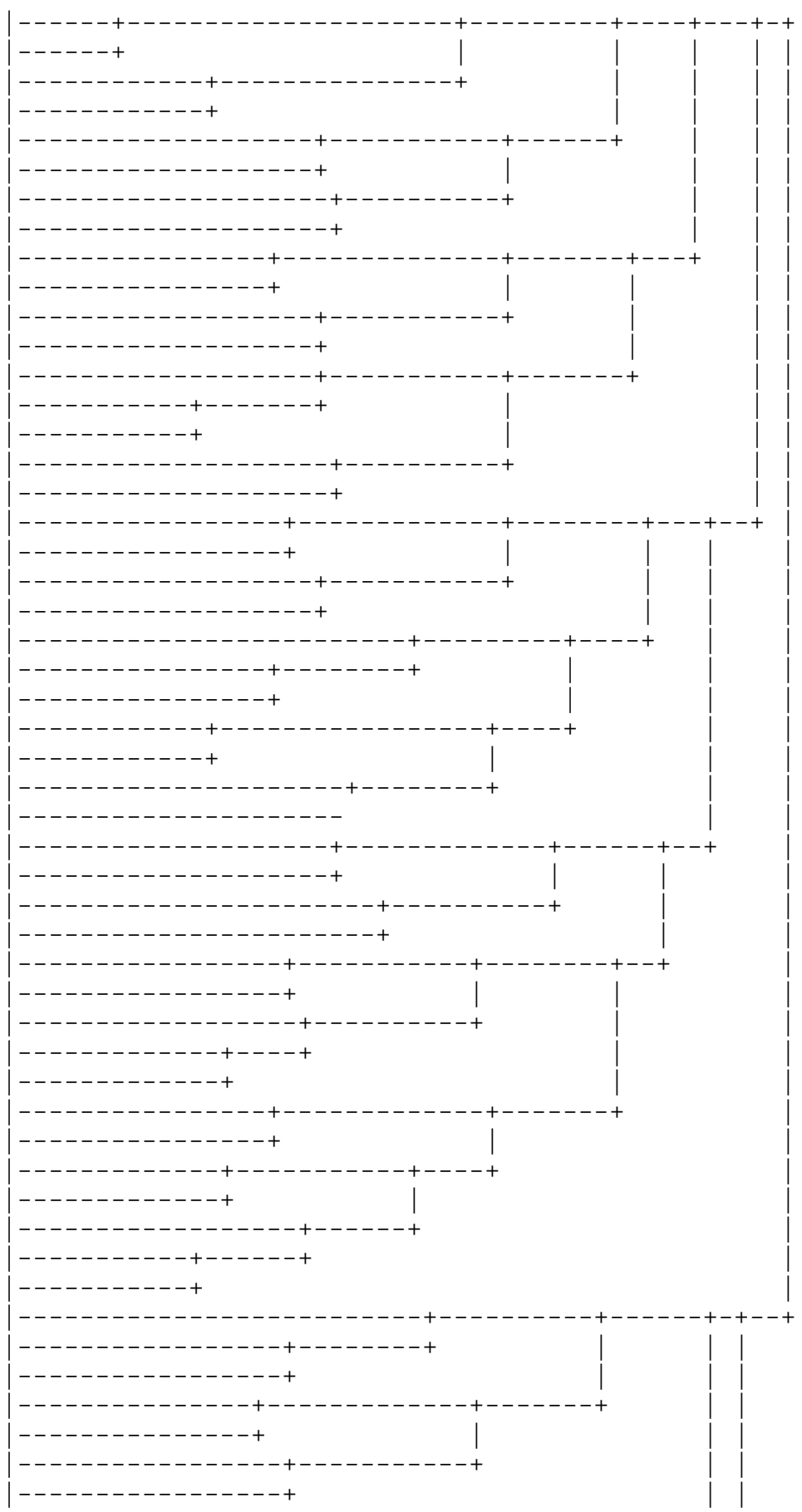


Strive

Encourage

Possess

Quality

Character

Essential

Consistent

Excellent

Continual

Constant

Drive

Attitude

Accomplish

Interpersonal

Artist

Craft

Attribute

Honest

Discipline

Self

Strength

Determination

Resilience

Compassion

Humility

Dedication

Require

Excel

Persevere

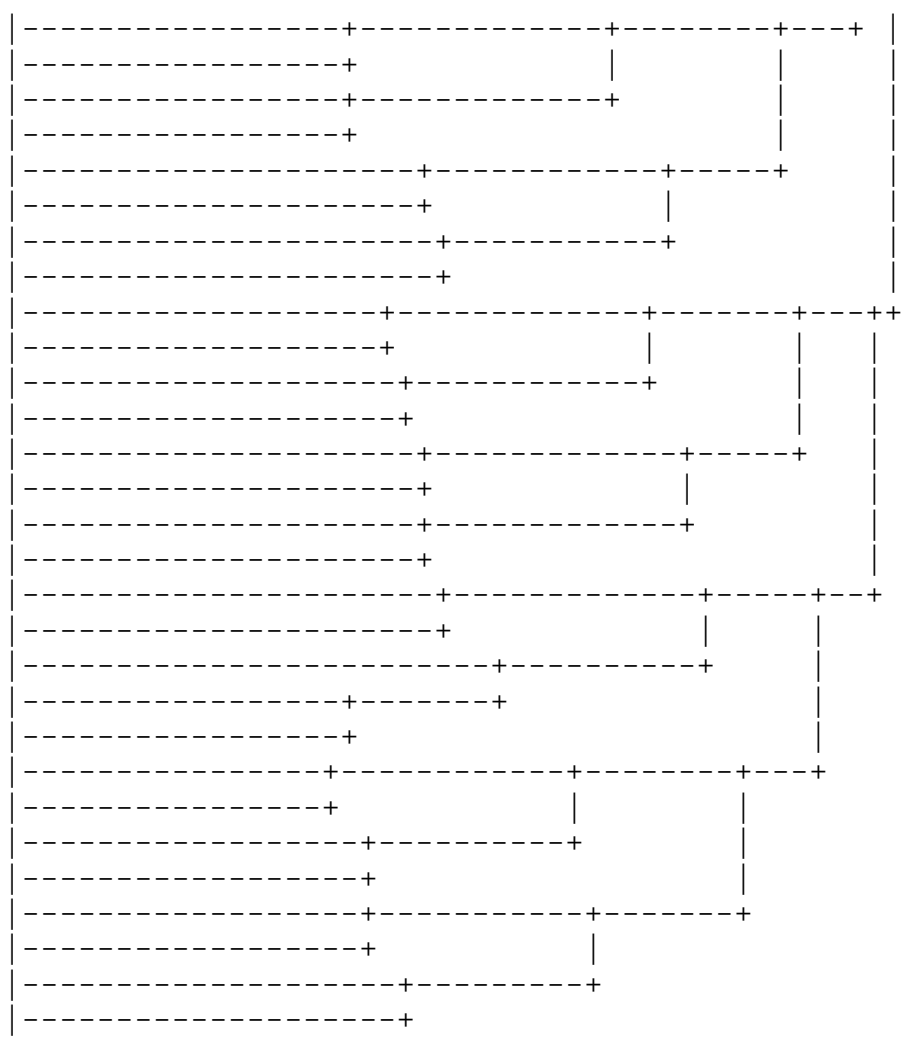

Class 4 - The art of surgery: This class focuses on the technical aspects of surgery, highlighting the precision, efficiency, and meticulousness of surgery.

Room
Attend
Schedule
Cardiac
Chief
Hernia
Repair
Cholecystectomy
Laparoscopy
Undergo
Appendectomy
Procedure
Whipple
Surgeries
Case
Scrubb
Minute
Resuscitate
Emergency
Fast
Pace
Proceed
Computed
Tomography
Anesthesia
Nurse
Admission
Discharge
Day

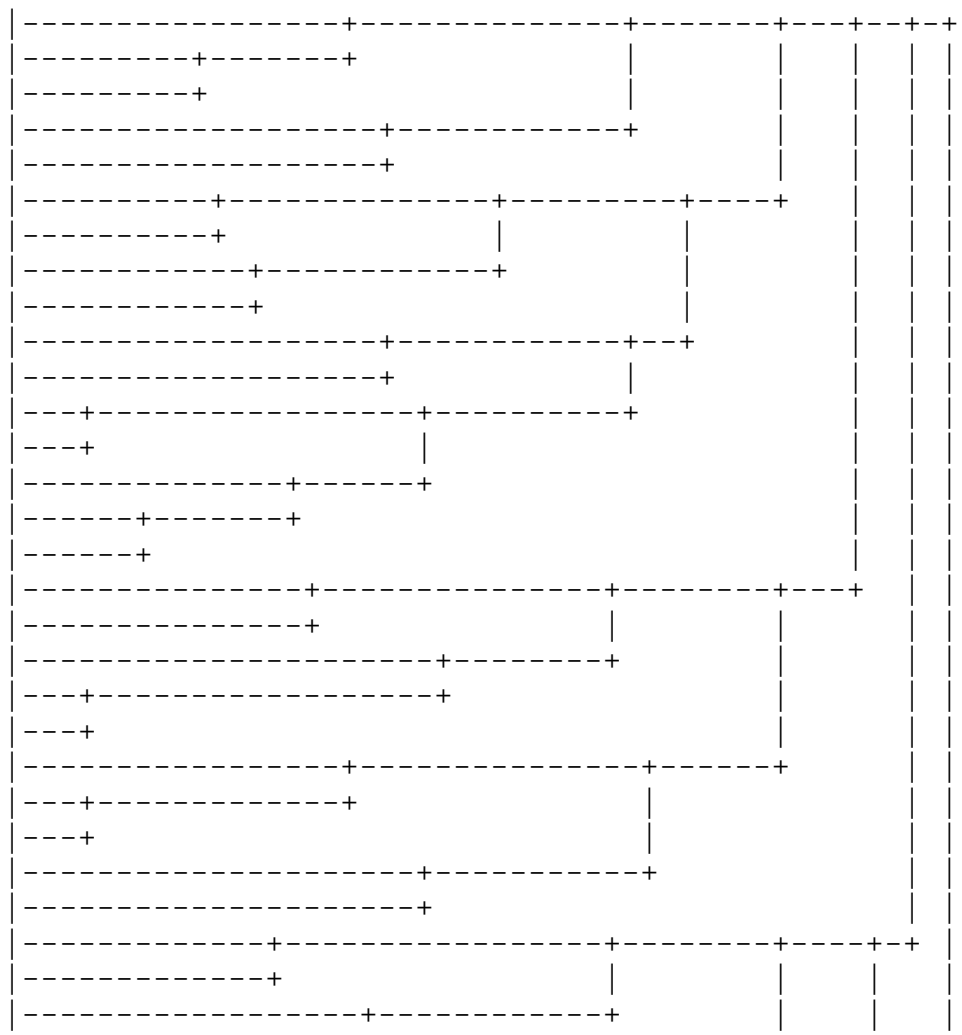




Hospital
Primary
Obstetrics
Gynecology
Morning
Fluid
Patient
Operating Room
Rotation
Operate
Witness
Op
Post
Pre
Month
Discuss
ICU
Subintern
Recover
Floor
Complication
Postoperative
Monitor
Follow
Bleed
Remove
Emergent
Abdominal
Aneurysm
Aortic
Pain
Woman
Impt Patient
Resect
Tumor
Abdomen
Liver
Severe
Bowel
Obstruct
Perforate
Sign
Exploratory
Laparotomy
Acute
Chronic
Managing
Clerkship
Colon
Consult
Evening
Page
Chest
Tube
Emergency_Dept
Man
Wrauma
Rush
Bay
Victim
Assist
Call

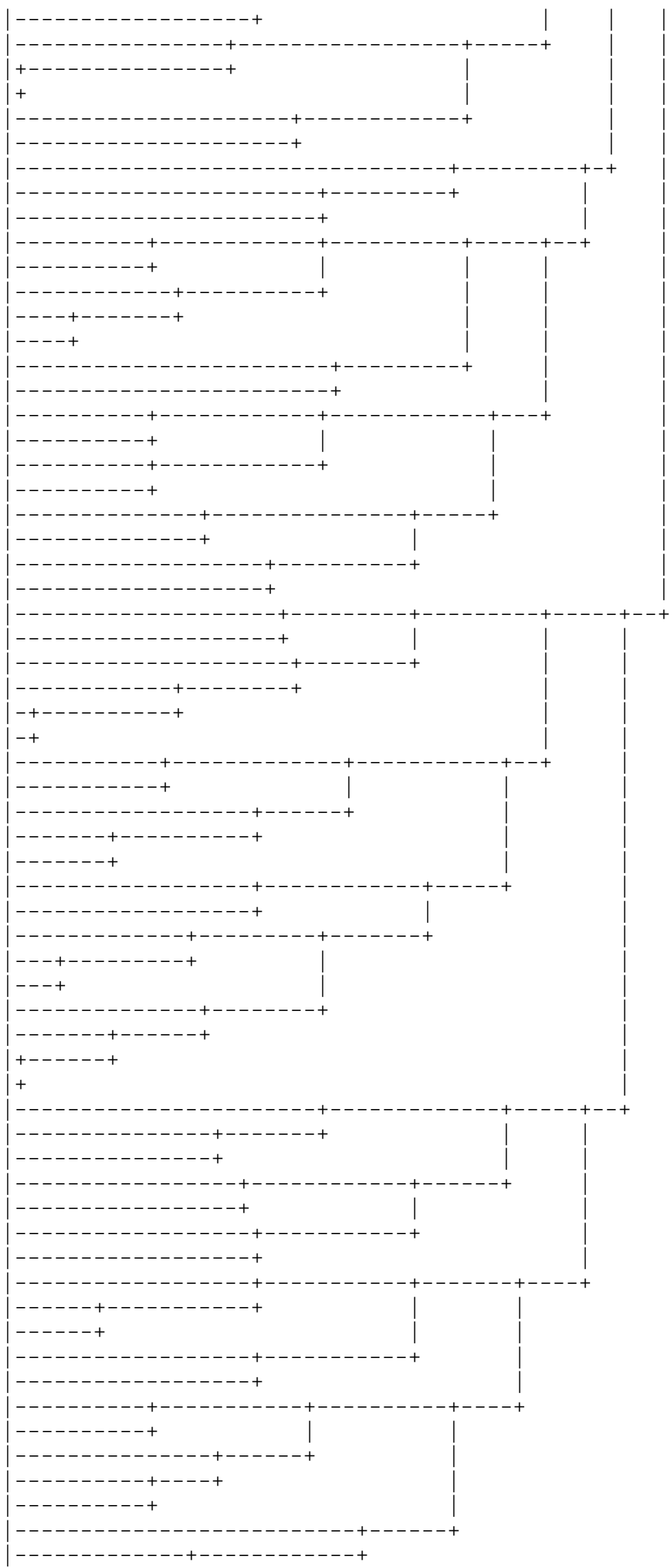


Night

| - . . . . + 